\title{
Letter to the Editor: What Is the Most Effective Exercise Intervention to Reduce Intermuscular Adipose Tissue in Older People?
}

I read the review by Waters ${ }^{1)}$ with great interest. I appreciate the authors' work to provide state-of-the-art knowledge on the effects of physical activity, weight loss, and obesity on intermuscular adipose tissue (IMAT) by comprehensively reviewing studies published in the last 10 years. I believe these findings will be of great help to draw new ideas from researchers in this field and to establish research directions.

The greatest change because of aging is the increase in body fat with a decrease in muscle mass. The increase in adipose tissue with age further reduces physical function in obese older adults, including those who are overweight, compared with physical function in older adults with normal weight. ${ }^{2,3)}$ The large cohort study of an older population suggested that the reduced density of quadriceps muscle induced a decreased knee extensor torque, which resulted in an increased vulnerability to falls and fractures. ${ }^{4)}$ Within the next few decades, it is predicted that approximately $40 \%$ of the population aged over 60 years will be classified as obese. ${ }^{5)}$ Therefore, investigating the effects of adipose tissue on physical function and disability will contribute to improving public health.

I agree that IMAT increases with aging and is affected by physical activity. The positive effects of exercise on fat reduction have been demonstrated. For example, longterm aerobic and anaerobic exercises reduce intramuscular fat in obese adults. ${ }^{6,7)}$ However, one study revealed that while muscle size increased after three weeks of interval training in obese young adults, there was no change in adipose tissue. ${ }^{8)}$ Thus, there is a need to clarify the effects of exercise on IMAT reduction. In particular, the type of exercise and the exercise intervention period should be investigated.

In addition, a higher IMAT results in reduced improve-

\section{Yunah Jeon}

Division of Sports and Health Science, Kyungsung University, Busan, Korea

Corresponding Author: Yunah Jeon, PhD

https://orcid.org/0000-0003-4484-4187

Division of Sports and Health Science, Kyungsung University, 309

Suyeong-ro, Nam-gu, Busan 48434, Korea

E-mail: jeonyn1219@gmail.com

Received: May 14, 2019

Accepted: May 25, 2019 ment in physical function compared with that in cases with lower IMAT, despite receiving the same exercise interventions. ${ }^{9,10)}$ These findings support the hypothesis of our ongoing research to investigate the effect of 12week resistance exercise training on single muscle fiber function in older adults with normal weight and obesity. To summarize the results of our analysis thus far, before the exercise intervention, higher isokinetic strength and greater contractility of single muscle fiber were observed in the obese group than in the normal weight group. These parameters improved in both groups after 12 weeks of resistance exercise; however, greater improvements were observed in the normal weight group than in the obese group. Although the mean body weight of the obese group decreased significantly after the exercise intervention, that of the normal-weight group did not. Delmonico et al. ${ }^{11)}$ reported that IMAT increased with age and was not related to body weight.

In summary, as demonstrated by previous studies, increased IMAT with aging shows a high likelihood of being reduced by physical activity. However, we cannot rule out the possibility that contradictory findings are obtained because of differences in exercise type and exercise duration and intensity. In addition, the possibility that IMAT can be reduced by physical activity regardless of weight loss requires consideration.

\section{CONFLICTS OF INTEREST DISCLOSURES}

The researcher claims no conflicts of interest.

\section{REFERENCES}

1. Waters DL. Intermuscular adipose tissue: a brief review of etiology, association with physical function and weight loss in older adults. Ann Geriatr Med Res 2019;23:3-8.

2. Woo J, Leung J, Kwok T. BMI, body composition, and physical functioning in older adults. Obesity (Silver Spring) 2007;15:188694.

3. Riebe D, Blissmer BJ, Greaney ML, Garber CE, Lees FD, Clark PG. The relationship between obesity, physical activity, and physical function in older adults. J Aging Health 2009;21:1159-78.

4. Frank-Wilson AW, Chalhoub D, Figueiredo P, Jónsson PV, Siggeirsdóttir K, Sigurdsson S, et al. Associations of quadriceps torque properties with muscle size, attenuation, and intramuscu- 
lar adipose tissue in older adults. J Gerontol A Biol Sci Med Sci 2018;73:931-8.

5. Ogden CL, Carroll MD, Fryar CD, Flegal KM. Prevalence of obesity among adults and youth: United States, 2011-2014. NCHS Data Brief 2015:1-8.

6. Durheim MT, Slentz CA, Bateman LA, Mabe SK, Kraus WE. Relationships between exercise-induced reductions in thigh intermuscular adipose tissue, changes in lipoprotein particle size, and visceral adiposity. Am J Physiol Endocrinol Metab 2008;295:E40712 .

7. Ryan AS, Ortmeyer HK, Sorkin JD. Exercise with calorie restriction improves insulin sensitivity and glycogen synthase activity in obese postmenopausal women with impaired glucose tolerance. Am J Physiol Endocrinol Metab 2012;302:E145-52.

8. Blue MNM, Smith-Ryan AE, Trexler ET, Hirsch KR. The effects of high intensity interval training on muscle size and quality in overweight and obese adults. J Sci Med Sport 2018;21:207-12.

9. Yoshida Y, Marcus RL, Lastayo PC. Intramuscular adipose tissue and central activation in older adults. Muscle Nerve 2012;46:8136.

10. Nicklas BJ, Chmelo E, Delbono 0, Carr JJ, Lyles MF, Marsh AP. Effects of resistance training with and without caloric restriction on physical function and mobility in overweight and obese older adults: a randomized controlled trial. Am J Clin Nutr 2015;101:991-9.

11. Delmonico MJ, Harris TB, Visser M, Park SW, Conroy MB, Velasquez-Mieyer P, et al. Longitudinal study of muscle strength, quality, and adipose tissue infiltration. Am J Clin Nutr 2009;90:157985. 